R. S. Manni

Nagoya Math. J.

Vol. 98 (1985), 99-107

\title{
ON THE DIMENSION OF THE VECTOR SPACE $C\left[\theta_{m}\right]_{4}$
}

\author{
RICCARDO SALVATI MANNI
}

\section{Introduction}

Let $\tau$ denote a point of the Siegel upper-half space $\subseteq g, z$ be a (column) vector in $C^{g}$ and $m$ a vector with $m^{\prime}$ resp. $m^{\prime \prime}$ in $Z^{g}$ as its first resp. second entry vectors, then the series

$$
\theta_{m}(\tau, z)=\sum_{p \in \boldsymbol{Z}^{g}} \boldsymbol{e}\left(\frac{1}{2}^{t}\left(p+\frac{m^{\prime}}{2}\right) \tau\left(p+\frac{m^{\prime}}{2}\right)+{ }^{t}\left(p+\frac{m^{\prime}}{2}\right)\left(z+\frac{m^{\prime \prime}}{2}\right)\right),
$$

in which $\boldsymbol{e}(t)=\exp \left(2 \pi(-1)^{1 / 2} t\right)$, represents an holomorphic function on the product $\widetilde{s}_{g} \times \boldsymbol{C}^{g}$ and it is called the theta function of characteristic $m$. It follows from the definition

$$
\theta_{m+2 n}(\tau, z)=(-1)^{t_{m^{\prime} n^{\prime \prime}}} \theta_{m}(\tau, z), \quad \theta_{m}(\tau,-z)=(-1)^{t_{m^{\prime} m^{\prime \prime}}} \theta_{m}(\tau, z) .
$$

Therefore up to sign there are $2^{2 g}$ theta functions and the function $\theta_{m}(\tau, z)$ is even or odd according as the characteristic $m$ is even or odd. If we put $z=0$ we get the so called Thetanullwerte $\theta_{m}=\theta_{m}(\tau, 0)$. It is well known that $\theta_{m} \neq 0$ if and only if $m$ is even, thus we can consider the graded ring $\boldsymbol{C}\left[\theta_{m}\right]$ generated over $C$ by the products of Thetanullwerte. Let us consider the complex vector space $C\left[\theta_{m}\right]_{k}$, i.e. the vector space spanned over $C$ by the monomials of degree $k$ in the Thetanullwerte, this has finite dimension over $C$.

Our results are:

i) $\operatorname{dim}_{C} C\left[\theta_{m}\right]_{4}=\left(\begin{array}{c}2^{g-1}\left(2^{g}+1\right)+3 \\ 4\end{array}\right)-\sum_{i=0}^{2} \mu_{i}\left(\nu_{i}-\pi_{i}\right)$

ii) All the quartic relations between the Thetanullwerte are consequence of the Riemann's theta-formula.

\section{The action of the symplectic group}

For any associative ring $R$ with 1 we shall denote by $S p(2 g, R)$ the

Received March 5, 1984. 
group of symplectic matrices of degree $2 g$ with coefficients in $R$. Its elements can be written as

$$
\sigma=\left(\begin{array}{ll}
a & b \\
c & d
\end{array}\right)
$$

in which $a, b, c, d$ are square matrices of degree $g$ with coefficients in $R$ satisfying $a^{t} d-b^{t} c=1_{g}, a^{t} b=b^{t} a$ and $c^{t} d=d^{t} c$.

We have denoted by $1_{g}$ the identity matrix of degree $g$. For any square matrix $s$ with $(i, j)$-th entry $s_{i j}$ we shall denote by $s_{0}$ the (column) vector $s_{i i}$ as its $i$-th entry.

Let $m$ be a vector in $Z^{2 g}$, we recall the formula

$$
\sigma \cdot m=\left(\begin{array}{rr}
d & -c \\
-b & -a
\end{array}\right)\left(\begin{array}{l}
m^{\prime} \\
m^{\prime \prime}
\end{array}\right)+\left(\begin{array}{l}
\left(c^{t} d\right)_{0} \\
\left(a^{t} b\right)_{0}
\end{array}\right)
$$

defines an action of $S p\left(2 g, \boldsymbol{F}_{2}\right)$ (i.e. $\left.S p(2 g, Z) \bmod 2\right)$ on the set $\left(\boldsymbol{F}_{2}\right)^{2 g}$.

For any $m, m_{1}, m_{2}, m_{3}$ in $Z^{2 g}$ we put

$$
\begin{aligned}
& e(m)=(-1)^{t_{m^{\prime} m^{\prime \prime}}}, \quad e\left(m_{1}, m_{2}\right)=(-1)^{t_{m_{1}^{\prime} m_{2}^{\prime \prime}-t_{m_{1}^{\prime \prime} m_{2}^{\prime}}}} \\
& e\left(m_{1}, m_{2}, m_{3}\right)=e\left(m_{1}\right) e\left(m_{2}\right) e\left(m_{3}\right) e\left(m_{1}+m_{2}+m_{3}\right)=e\left(m_{1}+m_{2}, m_{1}+m_{3}\right)
\end{aligned}
$$

We observe $e(m), e\left(m_{1}, m_{2}\right), e\left(m_{1}, m_{2}, m_{3}\right)$ depend only on $m, m_{1}, m_{2}$, $m_{3} \bmod 2$. We say $m$ is even or odd according as $e(m)=1$ or -1 .

For any $m, n$ in $\left(F_{2}\right)^{2 g}$ we shall denote by $m n$ the unique element of $\left(F_{2}\right)^{2 g}$ satisfying

$$
m n \equiv m+n \bmod 2
$$

With this law of composition $e(m, n)$ gives rise to a non-degenerate skew-symmetric bicharacter of $\left(\boldsymbol{F}_{2}\right)^{2 g} \times\left(\boldsymbol{F}_{2}\right)^{2 g}$.

A subspace $N$ of $\left(\boldsymbol{F}_{2}\right)^{2 g}$ is totally isotropic if $e(m, n)=1$ for every $m, n$ in $N$; in such a case the dimension $k$ of $N$ is at most equal to $g$. Moreover, fixing $k$, we have that the number of totally isotropic subspaces of dimension $k$ is given by

$$
\mu_{k}=\sum_{i=1}^{k}\left(2^{2(g-i+1)}-1\right) /\left(2^{k-i+1}-1\right)
$$

cf. [7].

We say that a coset $N m$ in $\left(F_{2}\right)^{2 g}$ is even or odd according as $e(m a)$ $=1$ or -1 for every $a$ in $N$. If such a "pure coset" exists than $N$ is necessarily totally isotropic. Furthermore if $k$ is the dimension of $N$ as 
above the number of pure coset is $2^{2(g-k)}$ and the number of even cosets is

$$
\nu_{k}=2^{g-k-1}\left(2^{g-k}+1\right)
$$

cf. [4] p. 213.

Let $m_{1}, \cdots, m_{r}$ be a sequence of (column) vectors in $Z^{2 g}$, we say that it is essentially independent if for any choice of $1 \leq i_{1}<\cdots<i_{2 k} \leq r$, where $k \geq 1$ we have

$$
\sum_{j=1}^{2 k} m_{i_{j}} \equiv 0 \bmod 2 .
$$

The point is that $e(m), e\left(m_{1}, m_{2}, m_{3}\right)$ or $e\left(m_{1}, m_{2}\right)$ and the property of being essentially independent are $S p(2 g, Z)$-invariant and they characterize the $S p(2 g, Z)$-orbits in $\left(F_{2}\right)^{2 g}$ in the following sense:

LEMMA 1 . Let $m_{1}, \cdots, m_{r} \bmod 2$ and $n_{1}, \cdots, n_{r} \bmod 2$ denote two sequences in $\left(\boldsymbol{F}_{2}\right)^{2 g}$ both with $r$ terms, then they are conjugate under the action of $S p\left(2 g, F_{2}\right)$ if and only if essentially independent subsequences correspond to each other and further

$$
e\left(m_{i}\right)=e\left(n_{i}\right) \quad \text { and } \quad e\left(m_{i}, m_{j}, m_{k}\right)=e\left(n_{i}, n_{j}, n_{k}\right)
$$

for all $1 \leq i \leq r$ and $1 \leq i<j<k \leq r$.

A proof of this lemma can be found in [4] p. 212.

Using this lemma we remark if $r=4$ and $e\left(m_{i}\right)=1$ for $i=1 \cdots 4$ we get exactly up to permutations $4,8,9$ orbits when $g$ is 1 , resp 2 , resp

\begin{tabular}{|c|c|c|}
\hline 1 & $m_{1}=m_{2}=m_{3}=m_{4}$ & $m_{i}={ }^{t}(0 \cdots 0) \quad i=1 \cdots 4$ \\
\hline 2 & $m_{1}=m_{2}=m_{3} \neq m_{4}$ & $\left(m_{1}, m_{4}\right)={ }^{t}\left(\begin{array}{lll}0 & \cdots & 0 \\
10 & \cdots & 0\end{array}\right)$ \\
\hline 3 & $m_{1}=m_{2} \neq m_{3}=m_{4}$ & $\left(m_{1}, m_{3}\right)={ }^{t}\left(\begin{array}{l}0 \cdots 0 \\
10 \cdots 0\end{array}\right)$ \\
\hline 4 & $\begin{array}{l}m_{1}=m_{2}, m_{i} \neq m_{j} \\
\quad \text { for } 2 \leq i<j \leq 4 \\
e\left(m_{2}, m_{3}, m_{4}\right)=-1\end{array}$ & 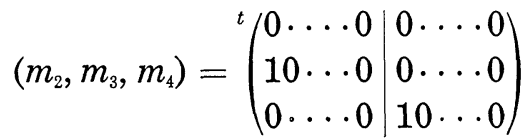 \\
\hline 5 & $\begin{array}{l}m_{1}=m_{2}, m_{i} \neq m_{j} \\
\quad \text { for } 2 \leq i<j \leq 4 \\
e\left(m_{2}, m_{3}, m_{4}\right)=1\end{array}$ & $\left(m_{2}, m_{3}, m_{4}\right)=\left(\begin{array}{l}0 \cdots \\
10 \cdots 0 \\
010 \cdots 0\end{array}\right)$ \\
\hline
\end{tabular}
$\geq 3$. We shall enumerate them from 1 to 9 and give below a characterization and a representative of each orbit 




We have denoted by $(\mid)$ the division of the first entry vector from the second.

\section{The dimension of $C\left[\theta_{m}\right]_{4}$}

We know that $S p(2 g, \boldsymbol{R})$ acts on $\mathfrak{S}_{g} \times \boldsymbol{C}^{g}$ as

$$
\sigma:(\tau, z)=\left((a \tau+b)(c \tau+d)^{-1},{ }^{t}(c \tau+d)^{-1} z\right) .
$$

We shall recall the transformation formula of theta functions

$$
\theta_{\sigma \cdot m}(\sigma \cdot(\tau, z))=k(\sigma) \operatorname{det}(c \tau+d)^{1 / 2} \boldsymbol{e}\left(\phi_{m}(\sigma)+\frac{1}{2}^{t} z(c \tau+d)^{-1} c z\right) \theta_{m}(\tau, z)
$$

in which

$$
\phi_{m}(\sigma)=-\frac{1}{2}\left({ }^{t} m^{\prime}{ }^{t} b d m^{\prime}+{ }^{t} m^{\prime \prime}{ }^{t} a c m^{\prime \prime}-2{ }^{t} m^{\prime} b c m-\left(a^{t} b\right)_{0}\left(d m^{\prime}-c m^{\prime \prime}\right)\right)
$$

We take $M=\left(m_{1}, \cdots, m_{r}\right)$ arbitrarily from $Z^{2 g}$ and put

$$
P(M)=\theta_{m_{1}} \cdots \theta_{m_{r}} .
$$

We say $P(M)$ a monomial of degree $r$ in the Thetanullwerte. Then, if for any $\sigma$ in $S p(2 g, Z)$ we put

$$
\sigma \cdot M=\left(\sigma \cdot m_{1}, \cdots, \sigma \cdot m_{r}\right)
$$

we get holomorphic functions $P(M)$ on $\mathbb{S}_{g}$ satisfying 


$$
P(\sigma \cdot M)(\sigma \cdot \tau)=k(\sigma)^{r} \operatorname{det}(c \tau+d)^{r / 2} e\left(\sum_{i=1}^{r} \phi_{m_{1}}(\sigma)\right) P(M)(\tau) .
$$

We know $P(M) \neq 0$ if and only if no $m$ is odd $1 \leq i \leq r$. We recall that if $m, a, b$ are arbitrary element of $Z^{2 g}$ then we have the Riemann's theta-formula for the Thetanullwerte:

$$
\theta_{m} \theta_{m+a} \theta_{m+b} \theta_{m-a-b}=2^{-g} \sum_{n \bmod 2} e(m, n) \theta_{n} \theta_{n+a} \theta_{n+b} \theta_{n-a-b}
$$

cf. [4] p. 141.

We shall convert the formula into one with elements of $\left(F_{2}\right)^{2 g}$ as subscripts of $\theta$. For that purpose we shall use the symbol

$$
(a, b, c)=e\left\{\frac{1}{2} \sum_{i=1}^{g}\left(a_{i}^{\prime} b_{i}^{\prime \prime} c_{i}^{\prime \prime}+a_{i}^{\prime \prime} b_{i}^{\prime} c_{i}^{\prime \prime}+a_{i}^{\prime \prime} b_{i}^{\prime \prime} c_{i}^{\prime}\right)\right\}
$$

in which $a, b, c$ are elements of $(Z)^{2 g}$ and $a_{i}^{\prime}$ resp $a_{i}^{\prime \prime}$ are the $i$-th coefficients of $a^{\prime}$ and $a^{\prime \prime}$. Then the theta-formula can be restated as follows

Lemma 2. If $m, a, b$ are in $\left(\boldsymbol{F}_{2}\right)^{2 g}$ then we have

$$
\begin{aligned}
& (m, a, a)(m, a, b)(m, b, b) \theta_{m} \theta_{m a} \theta_{m b} \theta_{m a b} \\
& \quad=2^{-g} \sum_{n \in\left(\boldsymbol{F}_{2}\right)^{2 g}} e(m, n)(n, a, a)(n, a, b)(n, b, b) \theta_{n} \theta_{n a} \theta_{n b} \theta_{n a b}
\end{aligned}
$$

A proof of this lemma can be found in [5].

From now on we shall assume $e(m)=1$. All relation of degree $k$ between the Thetanullwerte can be written as

$$
\sum_{j=1}^{n} c_{j} P\left(M_{j}\right)=0
$$

where $M_{j}=\left(m_{1}^{j}, \cdots, m_{k}^{j}\right) j=1 \cdots n$.

We say the relation (1) irreducible if

a) $c_{j} \neq 0$ for each $j$.

b) If $\sum_{i=1}^{n} c_{j_{i}} P\left(M_{j_{i}}\right)=0$ with $1 \leq j_{i} \leq n$ then $j_{i} \neq j_{k}$ for $i \neq k$ and $n=\bar{n}$.

c) $P\left(M_{j}\right) \neq P\left(M_{k}\right)$ if $j \neq k$.

Clearly we are interested only in studying irreducible relations. With this notations we have the following

LEMma 3. Let $\sum_{j=1}^{n} c_{j} P\left(M_{j}\right)$ denote a irreducible relation, the integral symmetric matrices of degree $2 g M_{j}{ }^{t} M_{j}$ and $M_{i}{ }^{t} M_{i}$ are related as follows:

$$
\begin{aligned}
& M_{j}{ }^{t} M_{j} \equiv M_{i}{ }^{t} M_{i} \bmod 2 \\
& \left(M_{j}{ }^{t} M_{j}\right)_{0} \equiv\left(M_{i}{ }^{t} M_{i}\right)_{0} \bmod 4 .
\end{aligned}
$$


Moreover (2) is preserved under the action of $\operatorname{Sp}(2 g, Z)$.

The proof of this lemma is similar to Lemma 2 in [3] and Lemma 2 in [6].

Now we are in the conditions of studying the quartic relations between the Thetanullwerte. They are completely described by the following

Lemma 4. There are not irreducible quartic relations in which occur monomials $P(M)$ with $m_{1}+m_{2}+m_{3}+m_{4} \neq \equiv 0(\bmod 2)$. Moreover the quartic relations are of the form:

(i ) $\sum_{m \text { even }} c_{m} \theta_{m}^{4}$

(ii) $\sum_{m \text { even }} c_{m} \theta_{m}^{2} \theta_{m n}^{2} \quad n \neq 0$ fixed

(iii) $\sum_{m \text { even }} c_{m} \theta_{m} \theta_{m a} \theta_{m b} \theta_{m a b} \quad a \neq 0, \quad b \neq 0, \quad a b \neq 0 a, b$ fixed.

Proof. Let us assume $\sum_{j=1}^{n} c_{j} P\left(M_{j}\right)=0$ and $m_{1}^{1}+m_{2}^{1}+m_{3}^{1}+m_{4}^{1} \not \equiv$ 0 mod 2 Applying the above lemma we can assume $M_{1}$ equal to one of the representative of the orbits $2,4,5,7,8,9$. We study only case 8 , because all the others are similar.

Thus we can assume

$$
M_{1}=\left(\begin{array}{cccc}
0 & 1 & 0 & 0 \\
\cdot & 0 & \cdot & \cdot \\
\cdot & \cdot & 0 & 0 \\
\hline \cdot & \cdot & 1 & 1 \\
\cdot & 0 & 1 \\
\cdot & \cdot & \cdot & 0 \\
0 & 0 & 0 & 0
\end{array}\right) .
$$

Then

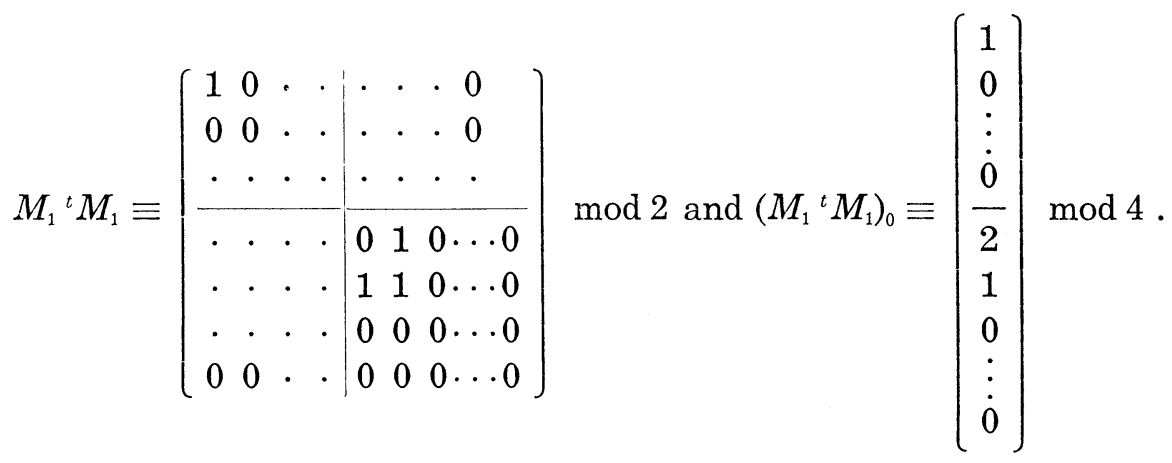


This implies that if $M_{1}$ and $M_{j}$ satisfy (2) then $M_{1}=M_{j}$, up to a permutation of the columns, thus $P\left(M_{1}\right)=P\left(M_{j}\right)$. The second part of the lemma can be proved in the same way.

We remark that the relations in (3) can be written as

$$
\sum_{N m \text { even }} c_{m} \prod_{n \in N m} \theta_{n}^{e}
$$

where $N$ is a fixed totally isotropic subspace of dimension $S=0,1,2$ and $2^{s} e=4$.

We want to compute the dimension of the $C$-vector space $C\left[\theta_{m}\right]_{4}$. Let $T_{g}$ be the $C$-vector space spanned by the $\theta_{m}^{4}$, we know

$$
\operatorname{dim}_{C} T_{g}=\left(2^{g}+1\right)\left(2^{g-1}+1\right) / 3=\pi_{0} \quad \text { cf. [8]. }
$$

Always from [8] we deduce that the dimension of the vector space spanned by $\theta_{m}^{2} \theta_{m a}^{2}$, a fixed and $a \neq 0$, is $\left(2^{g-1}+1\right) \cdot\left(2^{g-2}+1\right) / 3=\pi_{1}$ and the dimension of the vector space spanned by $\theta_{m} \theta_{m a} \theta_{m b} \theta_{m a b}, a, b$ fixed, $a \neq b, a \neq 0$, $b \neq 0$ is $\left(2^{g-2}+1\right) \cdot\left(2^{g-3}+1\right) / 3=\pi_{2}$. We recall we have $\mu_{1} \nu_{1}$ monomials of the form $\theta_{m}^{2} \theta_{m a}^{2}, a \neq 0$ such that $e(m)=e(m a)=1$, thus the dimension of the vector space spanned by these monomials is

$$
\mu_{1}\left(2^{g-1}+1\right)\left(2^{g-2}+1\right) / 3=\mu_{1} \pi_{1} .
$$

Moreover the monomials of the form $\theta_{m} \theta_{m a} \theta_{m b} \theta_{m a b}, a \neq 0, b \neq 0, a b \neq 0$ such that $e(m)=e(m a)=e(m b)=e(m a b)=1$ are $\mu_{2} \nu_{2}$, thus the dimension of the vector space spanned by these monomials is

$$
\mu_{2}\left(2^{g-2}+1\right)\left(2^{g-3}+1\right)=\mu_{2} \pi_{2} .
$$

Finally we know that all monomials $P(M)$ with $m_{1}+m_{2}+m_{3}+m_{4} \neq \equiv$ $\bmod 2$ are linearly independent and they span a vector space of dimension

$$
\left(\begin{array}{c}
2^{g-1}\left(2^{g}+1\right)+3 \\
4
\end{array}\right)-\sum_{i=0}^{2} \mu_{i} \nu_{i}
$$

If $\ell$ is a positive integer we shall denote by $\Gamma_{g}(\ell)$ the normal subgroup of $S p(2 g, Z)$ defined by $a \equiv d \equiv 1_{g}, b \equiv c \equiv 0 \bmod \ell$; we shall further denote by $\Gamma_{g}(\ell, 2 \ell)$ the subgroup of $\Gamma_{g}(\ell)$ defined by $\left(a^{t} b\right)_{0} \equiv\left(c^{t} d\right)_{0} \equiv$ $0 \bmod 2 \ell$.

It is a well known fact that

$$
\theta_{m}(\sigma \cdot \tau)=\operatorname{det}(c \tau+d)^{1 / 2} \theta_{m}(\tau)
$$

for every $\sigma$ in $\Gamma_{g}(4,8)$, then 


$$
P(M)(\sigma \cdot \tau)=\operatorname{det}(c \tau+d)^{2} P(M)(\tau)
$$

for $M=\left(m_{1}, m_{2}, m_{3}, m_{4}\right)$.

We have learned from J. Igusa [2] the following facts:

(a) (4) holds for every $\sigma$ in $\Gamma_{g}(4)$ if and only if

$$
\left(M^{t} M\right)_{0} \equiv 0 \bmod 2
$$

(b) (4) holds for every $\sigma$ in $\Gamma_{g}(2,4)$ if and only if

$$
\left(M^{t} M\right) \equiv 0 \bmod 2
$$

(c) (4) holds for every $\sigma$ in $\Gamma_{g}(2)$ if and only if $M$ satisfies (2).

Now let $\Gamma$ be a subgroup of $\operatorname{Sp}(2 g, Z)$ such that $\Gamma \supseteq \Gamma_{g}(4,8)$ then we can define $\left(C\left[\theta_{m}\right]_{4}\right)^{T}$ as the vector space spanned by the quartic polynomials invariant with respect to $\Gamma$. Moreover, always in [2] is proved that if $\Gamma \subseteq \Gamma_{g}(2)$ then a polynomial is invariant with respect to $\Gamma$ if each of the monomials occurring in the sum is invariant too. Thus we proved the following

THEOREM 1. (i ) $\operatorname{dim}_{C}\left(C\left[\theta_{m}\right]_{4}\right)^{\Gamma^{(2)}}=\pi_{0}$

(ii) $\operatorname{dim}_{C}\left(C\left[\theta_{m}\right]_{4}\right)^{\Gamma_{g^{(2,4)}}}=\pi_{0}+\mu_{1} \pi_{1}$

(iii) $\operatorname{dim}_{C}\left(C\left[\theta_{m}\right]_{4}\right)^{\Gamma^{(4)}}=\pi_{0}+\mu_{1} \pi_{1}+\mu_{2} \pi_{2}$

(iv) $\operatorname{dim}_{C} C\left[\theta_{m}\right]_{4}=q-\sum_{i=0}^{2} \mu_{i}\left(\nu_{i}-\pi_{i}\right)$

where $q=\left(\begin{array}{c}2^{g-1}\left(2^{g}+1\right)+3 \\ 4\end{array}\right)$.

We remark that $\left(C\left[\theta_{m}\right]_{4}\right)^{\Gamma} g^{(1)}=0$ since up to a constant factor an element of this vector space has to be the symmetrization of $\theta_{0}^{4}$ that is 0 .

Now we want to write all quartic relations using the Riemann's theta formula. Let us introduce the symmetric $2^{2 g}$ by $2^{2 g}$ matrix of signs

$$
K_{g}=\left(\begin{array}{ll}
K_{g}^{+} & L \\
{ }^{t} L & K_{g}^{-}
\end{array}\right)=e\left(m_{i}, m_{j}\right) \quad i, j=1 \cdots 2^{2 g}
$$

formed with respect to any fixed ordering of $m_{i}$ in $\left(\boldsymbol{F}_{2}\right)^{2 g}$ such that $K_{g}^{+}\left(K_{g}^{-}\right)$ is the matrix for $e(\cdot, \cdot)$ on the subset of the even (odd) characteristics.

We learned from [1] the following facts.

Lemma $5 . \quad K_{g}$ has two eigenspaces of dimension $l_{g}^{ \pm}=2^{g-1}\left(2^{g} \pm 1\right)$ with eigenvalues $\pm 2^{g}$ respectively, while $K^{ \pm}$has eigenspaces of dimension $\left(2^{g} \pm 1\right)\left(2^{g-1} \pm 1\right) / 3$ and $\left(2^{2 g}-1\right) / 3$ with eigenvalues $\pm 2^{g}$ and $\mp 2^{g-1}$ respectively.

If $K_{g}^{+} v=-2^{g-1} v$ for $v=\left(v_{i}\right)$ in $C^{l_{g}^{+}}$, then from the Riemann's theta formula we get 


$$
\begin{gathered}
\text { VECTOR SPACE }\left(C_{m}[\theta]\right)_{4} \\
\sum_{m \in K_{g}^{+}} v_{m}(m, a, a)(m, a, b)(m, b, b) \theta_{m} \theta_{m a} \theta_{m b} \theta_{m a b}=0 .
\end{gathered}
$$

Clearly the coefficients $v$ are defined on the even cosets $N m$ with $N$ $=\{a, a, b, a b\}$. Thus we can think of $v_{m}$ as a $-2^{g-k-1}$ eigenvector of $K_{g-k}^{+}$, where $k=\operatorname{dim} N$.

Now counting the relations that we obtain, we can see that these are all quartic relations between the Thetanullwerte. Thus we get

Proposition 1. All the quartic relations between the Thetanullwerte are consequence of the Riemann's theta formula.

\section{REFERENCES}

[1] J. Fay, On the Riemann-Jacobi formula, Göttingen Nachrichten, (1979), 61-73.

[2] I. Igusa, On the graded ring of theta constants, Amer. J. Math., 86 (1964), 219-246.

[ 3 ] - On Jacobi's derivative formula and its generalizations, Amer. J. Math., 102 (1980), 409-446.

[ 4 ] — Theta Functions, Grund, math. Wiss., 194, Springer-Verlag, 1972.

[ 5 ] —-, Schottky's invariant and quadratic forms, Christoffel Symposium, Birkhäuser Verlag (1981).

[6] - On the nullwerte of Jacobians of odd theta functions, preprint.

[ 7 ] A. Krazer, Lehrbuch der Thetafunktionen, B. G. Teubner, Leipzig, 1903.

[ 8 ] B. van Geemen, Siegel Modular forms vanishing on the Moduli space of curves, preprint (1983).

Dipartimento di Matematica

Università di Roma

Piazzale Aldo Horo

Roma, Italia 